\title{
Lectura y representación mental de textos expositivos en estudiantes de educación media
}

\author{
Reading and Mental Representation of Expository \\ Texts of High School Students
}

\author{
Geral Eduardo Mateus Ferro ${ }^{1}$ \\ Álvaro William Santiago Galvis ${ }^{2}$ \\ Myriam Cecilia Castillo Perilla ${ }^{3}$ \\ Luis Gonzalo Rodríguez López ${ }^{4}$
}

\section{Resumen}

Este artículo da cuenta de los resultados de una prueba de lectura realizada con el fin de caracterizar la representación mental de estudiantes de educación media, con base en la teoría de los niveles de representación planteados por Kintsch y Van Dijk (1983). La prueba hace parte del proyecto de investigación Formulación de una estrategia didáctica para la cualificación de la representación mental de textos expositivos en estudiantes de educación media, FHU-294-11.

\section{Palabras clave}

Lectura, representación mental, modelo de la situación, inferencias.

Abstract

This paper reports a finding from a reading test done in order to outline the mental representation of High School students, based on the theory of levels of representation proposed by Kintsch and Van Dijk (1983). The test is part of a research project, Developing a teaching strategy for the improvement of the mental representation of expository texts in High School students, FHU-294-11.

\section{Key words}

Reading, mind representation, theoretical situation model, inferences

Artículo recibido el 24 de enero de 2012 y aprobado el 19 de julio de 2012

1 Profesor Departamento de Lenguas, Universidad Pedagógica Nacional, Bogotá, Colombia. Miembro del grupo de investigación en Pedagogía, Lenguaje y Comunicación, Gipelec. Correo electrónico: gmateus@pedagogica.edu.co.

2 Profesor Departamento de Lenguas, Universidad Pedagógica Nacional, Bogotá, Colombia. Miembro del grupo de investigación en Pedagogía, Lenguaje y Comunicación, Gipelec. Correo electrónico: asantiago@pedagogica.edu.co.

3 Profesora Departamento de Lenguas, Universidad Pedagógica Nacional, Bogotá, Colombia. Miembro del grupo de investigación en Pedagogía, Lenguaje y Comunicación, Gipelec. Correo electrónico: mcastillo@pedagogica.edu.co

4 Profesor IED Álvaro Gómez, profesor Departamento de Lenguas, Universidad Pedagógica Nacional, Bogotá, Colombia. Correo electrónico: Ilrodríguez@gmail.com 
La lectura se ha constituido en área de interés tanto por su importancia en el proceso de formación del individuo, como por los problemas que los estudiantes tienen como lectores competentes, los cuales se evidencian en los distintos niveles del sistema educativo. Esto ha llevado a que se adelanten estudios e investigaciones con diferentes enfoques $\mathrm{y}$ desde distintas perspectivas, con el fin de, por un lado, entender el proceso como tal y, por el otro, formular propuestas didácticas, con las cuales se busca enfrentar y superar las deficiencias que presentan los estudiantes en lo que a la comprensión e interpretación lectoras respecta.

Esta deficiencia se debe a que los estudiantes no han logrado asumir, dado que tampoco han sido formados en ello, la lectura como proceso de orden cognitivo, sino que la toman como una actividad mecánica de simple decodificación de signos gráficos. Como consecuencia de esto, desconocen los diversos procesos que se ponen en marcha para adelantar las actividades de comprensión del código escrito a las que se ven enfrentados a diario, no solo en el ámbito escolar sino en su entorno sociocultural; por lo tanto, no logran planificar, regular ni evaluar dichas actividades, lo cual evidencia que no han desarrollado estrategias adecuadas que orienten la realización de cualquier ejercicio lector ${ }^{1}$.

En tal sentido, la psicología de la comprensión provee un marco para explicar las tareas cognitivas que tienen lugar en el proceso de lectura (Kintsch, 1988; Kintsch y van Dijk, 1983; Graesser, Millis y Zwaan, 1997). En general, desde esta perspectiva se asume la comprensión de textos como un proceso psicológico, el cual supone que más allá de la decodificación de signos escritos, es necesario reconstruir en la mente la red de significado que componen las ideas del texto, pues en la estructura superficial

1 Al respecto, cabe señalar que los factores asociados a los procesos tienen que ver con las actividades 0 acciones que adelanta el lector cuando intenta comprender lo que lee; estos procesos incluyen la atención, la codificación, la inferencia y la evocación, así como la regulación ejecutiva de los mismos a través del procesamiento metacognitivo, o el conocimiento sobre los procedimientos, y la evaluación de cómo se está procediendo. Esto lleva a plantear que la metacognición se constituye en un aspecto que se debe considerar al momento de analizar el proceso lector y, desde luego, a la hora de adelantar una intervención didáctica. de éste no se explicitan todos los elementos que se requieren para su comprensión. La tarea del lector consiste en crear y reconstruir informaciones que suplan los intersticios dejados por los signos gráficos. Comprender un texto implica, entonces, la creación de una representación compleja que integra tanto los conceptos explícitos del texto, como el conjunto de inferencias necesarias para establecer las referencias y asociaciones implícitas en él. Así mismo, este proceso requiere que la información aportada por el texto y la añadida por el sujeto se activen en la mente en determinados momentos (Kintsch, 1988; Kintsch y van Dijk, 1983).

El modelo de representación de niveles (Kintsch, 1988; Kintsch y van Dijk, 1983) da cuenta de estas características del procesamiento de los textos. Plantea que, como resultado del proceso de comprensión, se crea una representación organizada en niveles, que se constituye en una representación compleja que integra proposiciones en tres niveles y que, a su vez, representa las relaciones semánticas entre los significados que se activan en el proceso lector.

El primer nivel está constituido por la representación del conjunto de elementos percibidos directamente del texto; este conjunto se denomina código de superficie (o primer nivel). Este nivel de representación codifica aspectos literales como el vocabulario y la sintaxis oracional. El segundo nivel, el del texto base o base del texto, representa las proposiciones derivadas explícitamente del texto. En este nivel se almacenan solo los significados, de ahí que no se contemplen aspectos formales. De igual forma, en el proceso de construcción de la representación mental, los sujetos integran información propia, proveniente generalmente de la memoria de largo plazo (MLP), o llevan a cabo operaciones de transformación de la información, creando nuevos significados. Kintsch (1988) denomina a este nivel de representación, que integra la información explícita del texto con la aportada por el individuo, modelo de situación (tercer nivel). La nueva información, proveniente del lector, engloba los recuerdos, evocaciones y asociaciones construidas con base en la información representada en la 
base del texto, o la proveniente de los conocimientos previos (Graesser et al 19972).

De acuerdo con el modelo, todos los elementos que integran la representación del texto son formalizables en términos de proposiciones, las cuales corresponden a uno de los dos niveles: base del texto o modelo de situación. Podría decirse, entonces, que el propósito final del proceso de comprensión es recrear el significado de un texto. Para ello es preciso que interactúen los conocimientos del sujeto con los implicados en el texto; los conocimientos del sujeto incluyen tanto los lingüístico-estructurales necesarios para el reconocimiento de las estructuras de la lengua en los aspectos fonético, fonológico y sintáctico-como los conocimientos experienciales -propios de la socialización e incluyen aspectos pragmáticos- y los enciclopédicos -que hacen referencia a las posibles asociaciones directas e indirectas de las unidades léxicas (Kintsch, 1988; Kintsch y van Dijk, 1983).

En lo que compete al nivel de representación correspondiente al modelo de situación, es preciso anotar que una tarea clave tiene que ver con la generación de inferencias (Díaz y De Vega, 2003), puesto que éstas dan cuenta de la información que añade el lector a la que se presenta de forma explícita en el texto. Según León (2003), el estudio de las inferencias tiene mucha relevancia en la actualidad, por cuanto éstas se consideran el núcleo de la comprensión e interpretación de la realidad y uno de los pilares de la cognición humana. De este modo, en la comprensión del discurso escrito, el lector debe elaborar mentalmente una representación coherente de lo leído y para ello acude a las inferencias, las que, una vez activadas, establecen el puente entre la información leída y los conocimientos previos del sujeto.

En este sentido, se destaca el valor que tienen las inferencias para predecir conductas, para entender la realidad y para comprender mensajes abstractos. Por esta razón, cualquier información

2 Adicionalmente, otros autores (p.e. Zwaan, 1994; van Dijk, 1987) han anotado la existencia de otros niveles de representación, como el comunicativo, que hace referencia al contexto pragmático en el cual el texto está inmerso, y el nivel género textual.
Lectura y representación mental de textos expositivos en estudiantes de educación media Geral Eduardo Mateus Ferr Myriam Cecilia Castillo Perilla / Luis Gonzalo Rodríguez López

que se extrae de un texto y que no está expresada de forma directa, se puede considerar una inferencia. De esta manera, las inferencias que elabora el lector le permiten "cubrir los huecos" que trae el texto; en esta medida, las inferencias son producto del proceso cognitivo de inferir, por medio del cual el lector extrae información, tanto explícita como implícita, de los textos. Este proceso lleva a que el lector active conocimientos que no están en el texto, pero que sí forman parte de su saber y se encuentran almacenados en su memoria, o a que integre los conocimientos previos con los del texto, estableciendo relaciones y creando conocimientos "nuevos" (Díaz y De Vega, 2003; León, Escudero y Van den Broek, 2003; Trabasso y Magliano, 1996).

La activación de las inferencias en el proceso lector se da en función de dotar de sentido y coherencia lo que se lee; ahora bien, el tipo de inferencias que se activen dependerá de factores como el contexto y la disposición y acceso que se tenga al propio conocimiento. Siguiendo los planteamientos de León, se puede establecer una diferencia entre inferencias pragmáticas, las cuales se fundamentan en un saber compartido por personas pertenecientes a una misma cultura y tienen un valor probabilístico u opcional, y las inferencias lógicas, basadas en la aplicación de reglas formales (Díaz y De Vega, 2003; León, Escudero y Van den Broek, 2003; Trabasso y Magliano, 1996).

En los modelos de simulación sobre la comprensión de texto, se incluyen distintos tipos de inferencias pragmáticas y se afirma que el flujo de información depende del número y el tipo de inferencias que se deben hacer en el proceso de representación, construido en el curso de la comprensión. Tal representación debe ser coherente y las inferencias son determinantes al momento de establecer tanto la coherencia local, como la coherencia global del texto (Díaz y De Vega, 2003; León, Escudero y Van den Broek, 2003; Trabasso y Magliano, 1996).

En la literatura de la psicología del discurso se han propuesto variadas clasificaciones para dar cuenta de las inferencias (para un resumen de las taxonomías véase León, 2003, capítulo 2). No obstante, 
es reconocido el planteamiento de que al leer se realizan al menos tres tipos de inferencias básicas: explicativas, asociativas y predictivas (Trabasso y Magliano, 1996).

Las inferencias explicativas vinculan la información del texto con la información previa, estableciendo lazos causales, razón por la cual también se conocen como inferencias hacia atrás. Las inferencias asociativas brindan información sobre "las características, propiedades, relaciones y funciones de las personas, objetos o conceptos" (León, Escudero y Van den Broek, 2003, p. 167). Las inferencias predictivas proyectan consecuencias de la información que se ha ofrecido, por tal motivo se denominan también inferencias hacia adelante; al incluir consecuencias y proyecciones, algunos autores las han identificado como un tipo particular de inferencias explicativas (Zwaan y Brown, 1996).

Para efectos de adelantar la caracterización de las representaciones mentales de textos expositivos, se diseñó y aplicó una prueba de comprensión lectora ${ }^{3}$ a un grupo de estudiantes de grado $10^{\circ}$, de la institución educativa distrital Álvaro Gómez.

En relación con la prueba como tal, Aznar (1990, p. 47) plantea que existen cuatro tipos diferentes de pruebas que permiten la evaluación de las capacidades memorísticas de una persona. Éstas difieren entre sí según los propósitos que se definan y las maneras en que se obtienen los resultados. Estos tipos son: pruebas de recuerdo libre, pruebas de recuerdo con claves, pruebas de reconocimiento y pruebas de reconstrucción ${ }^{4}$.

3 El objetivo de caracterizar las representaciones mentales de esta población se enmarca en un proyecto más general, el proyecto FHU-294-11, que se formuló con el objetivo de diseñar y elaborar una propuesta de trabajo en lectura, a partir de los elementos que aporta la metacognición, en lo que tiene que ver con el uso de estrategias metacognitivas que pueden orientar el desarrollo y la optimización de esta habilidad en los estudiantes y, así, incidir en la cualificación de las representaciones mentales, esto es, de la comprensión lectora de este grupo. La prueba que se realizó corresponde a una de las tareas de la fase de diagnóstico del mencionado proyecto.

4 Las pruebas de recuerdo libre son aquellas en las que al sujeto se le presenta una lista de elementos y luego se le solicita que evoque todos los que pueda. En las pruebas de recuerdo con clave, al sujeto se le presenta uno de los elementos de la lista que presente relación con algún otro, debiendo recuperar la relación y el elemento que no se le ha ofrecido. Con las pruebas de reconstrucción se busca
Pérez (2005) y Montanero (2004) señalan que, junto con los test de verificación, las pruebas de recuerdo libre o evocación son las más empleadas en las investigaciones sobre comprensión lectora. De acuerdo con estos autores, en las investigaciones memorísticas, las pruebas de recuerdo libre consisten en recordar y presentar los elementos que conforman una lista; en investigación en comprensión lectora, las pruebas de recuerdo libre buscan que el lector evoque el mayor número de aspectos del texto que ha leído, sin tenerlo a la vista. La intención de estas pruebas consiste, entonces, en verificar qué cantidad de información retiene el lector, lo cual se realiza por medio de la producción de un texto ${ }^{5}$. Este texto permitirá evaluar la comprensión, dado que la producción de un mensaje sobre algo -en este caso un texto escrito- exige la comprensión previa de ese algo (Pérez, 2005, p. 126).

De acuerdo con lo anterior, las consideraciones que se exponen en este artículo corresponden a la presentación de los resultados que se obtuvieron de la aplicación de la prueba en cuestión, con base en los cuales se llevó a cabo el análisis de las representaciones mentales que los estudiantes elaboraron, teniendo como referente la teoría de los niveles de representación y los modelos de estructuras mentales ${ }^{6}$.

que la persona encuentre la dirección o el sentido de la relación entre dos elementos. Finalmente, la prueba de reconocimiento, la menos exigente memorísticamente, consiste en mostrar al sujeto diferentes elementos pertenecientes y no pertenecientes a la lista inicial y éste deberá reconocer cuáles hacen parte del grupo. En lo que a comprensión lectora atañe, estos tipos de pruebas se han empleado en diferentes investigaciones, de tal forma que existen pruebas de recuerdo libre visibles en estrategias como los resúmenes y los protocolos de recuerdo, pruebas de recuerdo con claves en los test de comprensión con preguntas de múltiple respuesta, pruebas de reconstrucción en la realización y evaluación de esquemas y gráficos, y pruebas de reconocimiento basadas en estrategias como las listas de verdadero/falso.

5 Según Montanero (2004, p. 420), la evaluación de este procedimiento presenta diferentes posibilidades, dentro de las cuales se destaca el contraste proposicional. Por medio de este procedimiento, el texto objeto de lectura y el texto que produce el lector se dividen en unidades proposicionales y, con base en ello, se adelanta una comparación. Este contraste tiene como objetivo encontrar qué aspectos relevantes del texto leído aparecen en el que produce el lector, así como la presencia y tipo de informaciones nuevas, aspectos estos que ofrecen datos sobre el proceso inferencial y de comprensión del lector.

6 Las teorías de los modelos mentales corresponden a "teorías sobre la comprensión que involucran la representación de situaciones 
Específicamente se espera determinar qué tipo de información constituye la representación en los niveles base del texto y modelos de situación: ¿qué porcentaje de la información de las representaciones proviene del texto y cuál es añadida por los estudiantes? ¿Esta información añadida por los estudiantes es consistente con el contenido del texto? ¿Cuál es el reparto de las inferencias en el nivel modelo de situación 7 ?

\section{Método}

Se aplicó una prueba de recuerdo libre a 20 estudiantes del grado décimo de la institución educativa distrital Álvaro Gómez ${ }^{8}$, provenientes de los estratos uno y dos, y cuyas edades oscilan entre los 14 y los 16 años de edad. Estos estudiantes presentan, en su mayoría, un desempeño escolar básico en el área de humanidades, el cual, según el sistema de evaluación adoptado por la institución, oscila en un rango que va de 30 a 39 puntos sobre 50 .

Para llevar a cabo esta prueba se utilizó como insumo el texto informativo La evolución de las especies (tomado de Vidal-Abarca, Gilabert y Abad, 2002). La realización de la prueba se hizo en una sesión colectiva de trabajo de una hora. La actividad se dividió en tres momentos: instrucción, lectura y escritura. En el primero, el docente encargado de

descritas en el texto" (Pinzás, 2003, p. 85). Según estas teorías, el lector, además de procesar proposiciones, construye un modelo mental que "consiste de símbolos mentales o señas organizadas en una estructura que pinta la situación descrita en el texto" (Pinzás, p. 85). De esta forma, los lectores pueden procesar un texto, ya sea a través de la representación proposicional o creando un modelo mental; esto está determinado por el tipo de texto y el propósito de la lectura.

7 Dado que algunos de los antecedentes investigativos plantean resultados divergentes en lo que tiene que ver con las inferencias (León, Escudero y Van den Broek, [2003], por ejemplo, hallaron que los textos expositivos suelen generar más elaboraciones y explicaciones para llevar a cabo la integración entre el contenido del texto y el conocimiento previo, mientras que Narváez, Van den Broek y Ruíz [1999], en un estudio con protocolos verbales, encontraron que los participantes generaron un mayor número de inferencias asociativas que explicativas y predictivas al leer textos expositivos), se espera determinar cuál es el patrón de distribución de los tres tipos de inferencia.

8 Esta institución educativa está ubicada en la localidad 11 (Suba) de Bogotá y ofrece el ciclo completo de la educación básica primaria, básica secundaria y educación media en la modalidad de bachiIlerato académico. Cuenta con dos sedes y atiende una población promedio de 2500 estudiantes en jornadas mañana y tarde.
Lectura y representación mental de textos expositivos en estudiantes de educación media Geral Eduardo Mateus Ferr Myriam Cecilia Castillo Perilla / Luis Gonzalo Rodríguez López

aplicar la prueba ofreció las instrucciones necesarias para hacer el ejercicio, en términos de explicar en qué consistía, cómo desarrollarlo y cuánto tiempo se disponía para ello; asimismo, resolvió las inquietudes del caso. Una vez presentadas las instrucciones, se pasó al momento de la lectura, para lo cual se le entregó a cada estudiante el texto en cuestión y se estableció un tiempo de 20 minutos para leerlo. Después de agotar este plazo, se recogió el texto, se le entregó a cada estudiante una hoja en blanco y se le solicitó que en ella escribiera toda la información que recordara del texto que acababa de leer; para esto se determinó un tiempo de 15 minutos.

Para efectos de identificar y caracterizar las representaciones mentales de los estudiantes, se analizaron las pruebas contrastándolas con el texto base $^{9}$, con el fin de determinar la correspondencia del texto con las pruebas y, así, poder establecer la relación entre la representación mental y el nivel de comprensión del texto. Para adelantar esta tarea se diseñó un formato, basado en las propuestas de Zwaan y Brown (1996) y de Narváez, Van den Broek y Barrón-Ruiz (1999), que permite distinguir las proposiciones de los textos que produjeron los estudiantes, según se asuman como parte de la base del texto o del modelo de situación. En la tabla 1 se presentan las categorías de análisis que se establecieron.

Como se puede observar, la rejilla se encuentra dividida en tres columnas: proposiciones, base del texto y modelo de la situación. En la primera columna se registran las proposiciones, de acuerdo con la segmentación que se realizó previamente. La columna base del texto hace referencia a si la proposición corresponde a alguna de las proposiciones del texto base, correspondencia que puede ser literal o transformada. Por su parte, el modelo de la situación está conformado por las inferencias, clasificadas en asociativas,

9 En el anexo se encuentra el análisis por proposiciones realizado al texto original. Para el análisis, el grupo de investigadores segmentó una a una las oraciones del texto y las formuló de modo que fueran explícitas las proposicionales. En algunos casos fue necesario aplicar reglas de reconstrucción para explicitar el significado completo de algunas oraciones, debido a los fenómenos de elipsis propios de la redacción. Cada investigador hizo un análisis individual y luego se consolidó un solo análisis en el que se resolvieron desacuerdos semánticos. 
explicativas y predictivas, y por las evaluaciones; para las inferencias asociativas y explicativas, a su vez, se establece si éstas surgen de la base del texto o de los conocimientos previos del lector y si son consistentes (C) o inconsistentes (INC) con el texto base; en cuanto a las predicciones, se establece si son consistentes o no con el texto en cuestión; entre tanto, para las evaluaciones, se establece si corresponden al contenido (C), a la forma $(\mathrm{F})$, o son comentarios sobre la tarea $(\mathrm{T}) \mathrm{o}$ el texto en general (CO).

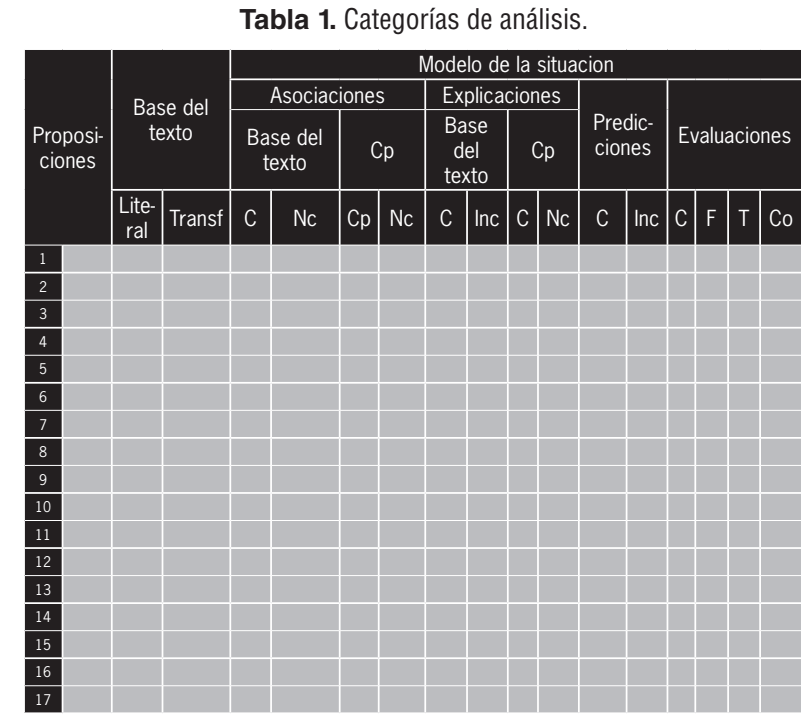

La codificación de cada una de las pruebas fue llevada a cabo por los miembros del proyecto de investigación de manera individual y luego cada codificación fue evaluada por otro miembro del grupo. Los desacuerdos fueron sometidos a discusión por todos los miembros del equipo investigador. Finalmente, se procesó la información resultante de la codificación y análisis, y se sometieron los datos a análisis estadísticos ${ }^{10}$.

\section{Resultados}

Tal como se expuso, uno de los objetivos de la investigación consistió en caracterizar la representación mental que los estudiantes generaron con base en la lectura del texto La evolución de las especies; para tal fin se aplicó una prueba de recuerdo libre. De los resultados de ésta, se tomaron como base del análisis las medias de cada una de las categorías y

10 Para el análisis estadístico de la información procesada a partir de las pruebas, se empleó el paquete estadístico SPSS. Se aplicaron la Prueba T para comparar las medias y Anova para muestras relacionadas según las características de las variables. subcategorías que se definieron. Sin embargo, para contrastar los resultados de los estudiantes con los juicios de expertos ${ }^{11}$, se hizo una prueba adicional, consistente en pedirle a un grupo de profesionales que subrayaran en el texto las ideas que consideraban como las más importantes; esto con el fin de tener un punto de referencia en relación con la información relevante del texto y establecer en qué medida los estudiantes centraron su atención en la información clave para la comprensión del mismo. En esta medida, primero se presentan los resultados de la prueba de recuerdo y luego el contraste de éstos con los obtenidos en las pruebas desarrolladas por los expertos.

Tabla 2. Frecuencia media del tipo de información según los niveles de representación.

\begin{tabular}{|c|c|c|c|c|}
\hline \multirow{3}{*}{$\begin{array}{l}\text { Base del } \\
\text { texto }\end{array}$} & Literal & 2 & & \\
\hline & $\begin{array}{l}\text { Transforma- } \\
\text { da }\end{array}$ & \multicolumn{3}{|l|}{3,85} \\
\hline & $\begin{array}{l}\text { Total base } \\
\text { del texto }\end{array}$ & \multicolumn{3}{|l|}{4,05} \\
\hline \multirow{18}{*}{$\begin{array}{l}\text { Modelo de } \\
\text { situación }\end{array}$} & \multirow{4}{*}{$\begin{array}{l}\text { Asociacio- } \\
\text { nes }\end{array}$} & \multirow{2}{*}{$\begin{array}{l}\text { Base del } \\
\text { texto }\end{array}$} & $\begin{array}{l}\text { Consisten- } \\
\text { tes }\end{array}$ & 6,45 \\
\hline & & & $\begin{array}{l}\text { No consis- } \\
\text { tentes }\end{array}$ & 5,55 \\
\hline & & \multirow{2}{*}{$\begin{array}{l}\text { Conoci- } \\
\text { miento pre- } \\
\text { vio }\end{array}$} & $\begin{array}{l}\text { Consisten- } \\
\text { tes }\end{array}$ & 2,25 \\
\hline & & & $\begin{array}{l}\text { No consis- } \\
\text { tentes }\end{array}$ & 3,35 \\
\hline & \multicolumn{3}{|c|}{ Total asociaciones } & 17,6 \\
\hline & \multirow{4}{*}{$\begin{array}{l}\text { Explicacio- } \\
\text { nes }\end{array}$} & \multirow{2}{*}{$\begin{array}{l}\text { Base del } \\
\text { texto }\end{array}$} & $\begin{array}{l}\text { Consisten- } \\
\text { tes }\end{array}$ & 0,65 \\
\hline & & & $\begin{array}{l}\text { No consis- } \\
\text { tentes }\end{array}$ & 1,7 \\
\hline & & \multirow{2}{*}{$\begin{array}{l}\text { Conoci- } \\
\text { miento pre- } \\
\text { vio }\end{array}$} & $\begin{array}{l}\text { Consisten- } \\
\text { tes }\end{array}$ & 0,35 \\
\hline & & & $\begin{array}{l}\text { No consis- } \\
\text { tentes }\end{array}$ & 0,8 \\
\hline & \multicolumn{3}{|c|}{ Total explicaciones } & 3,5 \\
\hline & \multirow{2}{*}{$\begin{array}{l}\text { Prediccio- } \\
\text { nes }\end{array}$} & \multicolumn{2}{|l|}{ Consistentes } & 0,65 \\
\hline & & \multicolumn{2}{|c|}{ No consistentes } & 0,45 \\
\hline & \multicolumn{3}{|c|}{ Total predicciones } & 0,85 \\
\hline & \multicolumn{3}{|c|}{ Total inferencias } & 22,2 \\
\hline & \multirow{3}{*}{$\begin{array}{l}\text { Evaluacio- } \\
\text { nes }\end{array}$} & \multicolumn{2}{|l|}{ Contenido } & 0,75 \\
\hline & & \multicolumn{2}{|l|}{ Forma } & 0 \\
\hline & & \multicolumn{2}{|l|}{ Tarea } & 0,1 \\
\hline & \multicolumn{3}{|c|}{ Total modelo de situación } & 23,05 \\
\hline
\end{tabular}

11 Los sujetos expertos son profesores universitarios de áreas como lingüística, literatura, pedagogía, filosofía. 


\section{Prueba de recuerdo}

Como resultado del análisis de la prueba de recuerdo, se halló que la media de proposiciones que integran las representaciones generadas por los estudiantes en la prueba es de 27,1 ( $\mathrm{SD}=9,6)$, que equivale al $28 \%$ del total de 94 proposiciones que constituyen el texto de prueba. Es decir que del $100 \%$ de las proposiciones que integran el texto de la prueba, el recuerdo de la información es cercano a un tercio. El desglose del tipo de información registrado en las representaciones se presenta en la tabla 2, especificando a qué nivel -base del texto o modelo de situación- corresponde la información, así como las subcategorías que integran cada nivel. Consecuentemente, los resultados que se exponen enseguida están organizados en torno a los dos niveles de representación (base del texto y modelo de situación), contrastando las informaciones correspondientes a cada uno.

En cuanto a la relación base del texto/modelo de situación, el contraste entre la frecuencia media de la información de cada nivel revela que, de manera significativa $(t=-8,4, p<0,05)$, se ofrece más información correspondiente al modelo de situación que a la base del texto. La Figura 1 ilustra estas diferencias.

Figura 1. Nivel de representación.

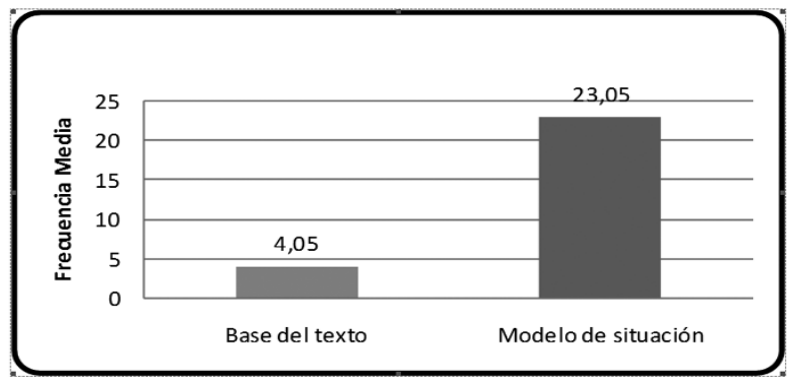

\section{Base del texto}

De acuerdo con lo expuesto, la información del nivel base del texto corresponde a aquella consignada de manera explícta en el texto leído, a la cual los estudiantes hacen referencia de manera literal o transformada. Entre tanto, la información del nivel modelo de situación es aquella que añade el lector y que tiene que ver tanto con las inferencias como
Lectura y representación mental de textos expositivos en estudiantes de educación media Geral Eduardo Mateus Ferr Myriam Cecilia Castillo Perilla / Luis Gonzalo Rodríguez López

con las evaluaciones. Así las cosas, se evidencia que las representaciones generadas se guían más por la información aportada por los estudiantes que por la del texto base. No obstante, se podría señalar que dicha información la genera la lectura del texto; aquí se debe tener en cuenta, entonces, la calidad de la información en términos del grado de coincidencia con la que presenta el texto trabajado.

En cuanto a la base del texto, en lo que tiene que ver con la relación información literal/información transformada, al recurrir a una prueba de recuerdo, se esperaba que la información coincidente con la de la base del texto se almacenara en mayor medida en un formato proposicional, sin tener las características formales del nivel de superficie. En efecto, los resultados demuestran que la media de información transformada (p. ej. a través de sinónimos) del nivel base del texto es 3,85 , la cual es significativamente mayor $(\mathrm{t}=-4,038, \mathrm{p}<0,05)$ que la literal, que fue del 0,2 . La proposición más recordada literalmente es el título -La evolución de las especies-, lo que era de esperar si se tiene en cuenta su posición sobresaliente en el texto. Un ejemplo de proposición transformada es: "Si a un animal le faltaba una pata toda su descendencia saldrian pero con las dos patas", que retoma lo expresado literalmente en el texto del siguiente modo: "Un animal al que le falte una pata no tiene por qué producir descendientes sin esa pata" (Proposición $\mathrm{N}^{\circ}$ 38. Cfr. el anexo).

Figura 2. Base del texto.

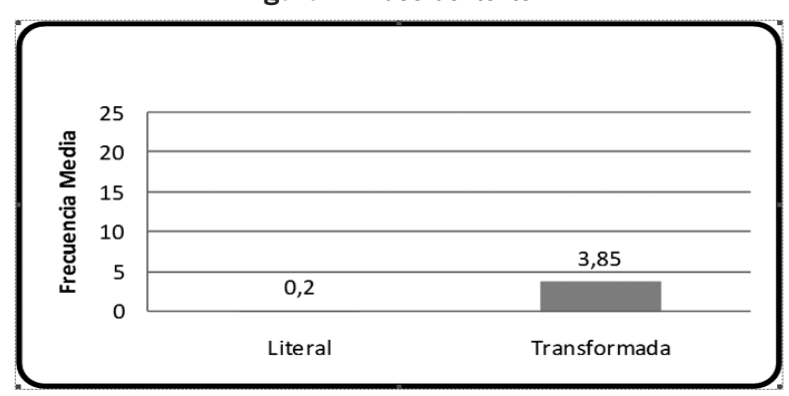

\section{Modelo de situación}

En relación con el modelo de situación, los resultados muestran que el modelo construido está compuesto en su mayoría por inferencias (93\%) y en menor medida por evaluaciones (7\%). La Figura 
3 presenta la frecuencia media de los tres tipos de inferencia y de las evaluaciones que integran el modelo de situación de las pruebas realizadas con el texto La evolución de las especies.

Figura 3. Modelo de situación.

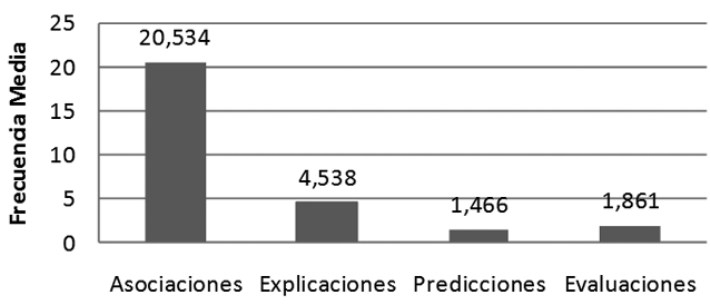

Los análisis estadísticos (Anova) muestran un efecto del factor 'Inferencia' $(\mathrm{F}(2,18)=28,181$, $\mathrm{p}=0,000$ ). Los contrastes entre los tipos de inferencias revelan diferencias significativas entre las inferencias asociativas y las explicativas $(\mathrm{p}<0,05)$ $y$ predictivas $(p<0,05)$. El contraste entre las inferencias explicativas y predictivas no es significativa $(\mathrm{p}>0,05)$.

De otro lado, en el análisis hecho a las proposiciones del nivel modelo de situación, no solo se consideró el tipo de información sino también el origen de ésta. En cada proposición se analizó si proviene de la información que aparece en la base del texto, añadiendo información adicional, es decir, a través de inferencias, o si las proposiciones se fundan en el conocimiento previo de los estudiantes. La Figura 4 ilustra las diferencias entre la información proveniente de la base del texto y del conocimiento previo. Las pruebas estadísticas muestran que la fuente de información más frecuente en el modelo de la situación es la base del texto, por encima del conocimiento previo $(\mathrm{t}=3,04, \mathrm{p}<0,05)$.

Figura 4. Fuente de la información del Modelo de situación.

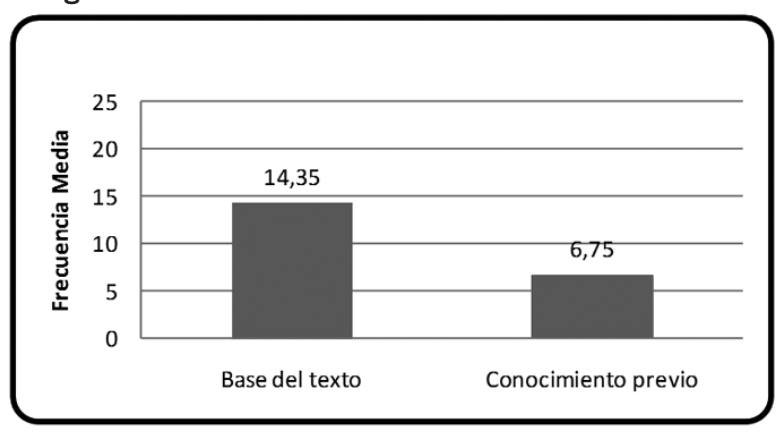

Figura 5. Calidad de la información.

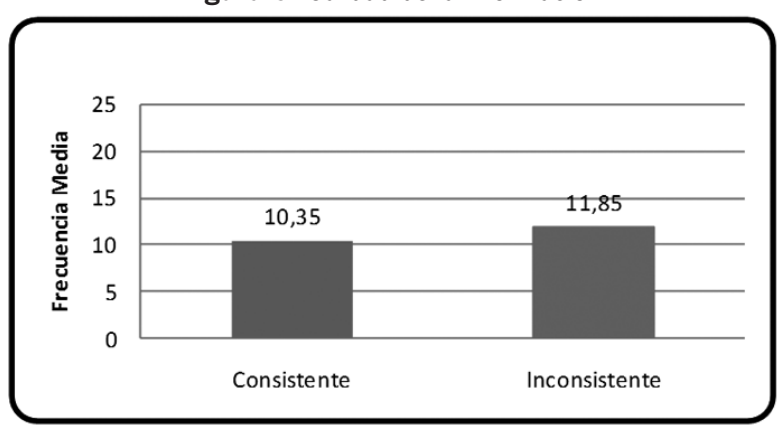

Adicionalmente, en el análisis de la proposiciones se estableció la distinción entre la información consistente (p. ej. "Los animales van cambiando al paso del tiempo") o la información inconsistente, con la presentada en el texto original (p. ej. "Los animales que fueron cambiando como el elefante, que primero era pequeño, y casi no tenía trompa, ahora es grande y tiene una enorme trompa"). Así las cosas, la información consistente es aquella que mantiene el significado expuesto en el texto, mientras que la inconsistente corresponde a aquellas proposiciones que van en contraposición con el conocimiento que presenta el texto base, en la medida que lo alteran o tergiversan. Como se puede apreciar en la Figura 5, la información inconsistente presenta mayor índice de frecuencia con respecto a la consistente.

Figura 6. Calidad de la información (M de S).

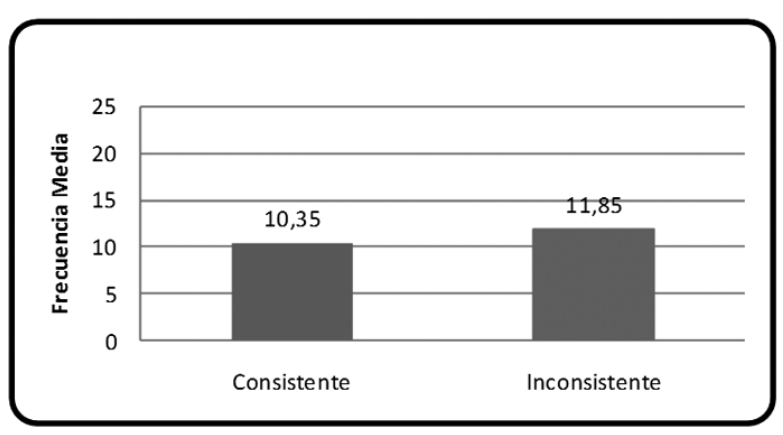

También se analizó cómo la calidad de la información (consistente vs. inconsistente) se relaciona con la comprensión de la información fundada en la base del texto o en el conocimiento previo. Como puede notarse en los dos ejemplos anteriores, el primero está vinculado a la base del texto, en tanto que el segundo claramente se fundamenta en el conocimiento previo del estudiante. La Figura 6 muestra 
que la mayor fuente de información consistente proviene de la base del texto frente al conocimiento previo. En este caso, las diferencias son estadísticamente significativas $(\mathrm{t}=3,231, \mathrm{p}<0,05)$.

Del mismo modo, la mayor fuente de información inconsistente (ver Figura 7) se relaciona con la base del texto. Sin embargo, en este caso las diferencias no fueron estadísticamente significativas.

Figura 7. Distribución de la información inconsistente (M de S).

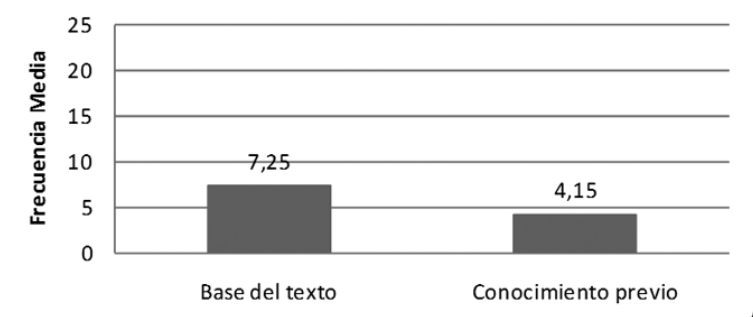

Con respecto a la calidad de la información, se obtuvieron estos resultados:

- Inferencias asociativas. Las figuras 8 y 9 presentan los resultados específicos de las inferencias asociativas.

Figura 8. Asociaciones (Conocimiento previo).

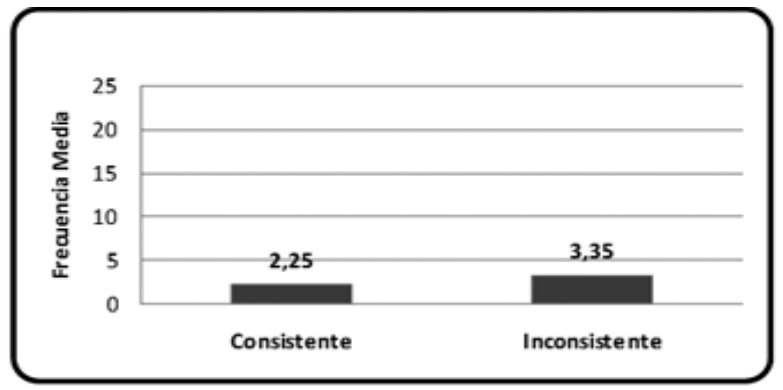

Figura 9. Asociaciones (Base del texto).

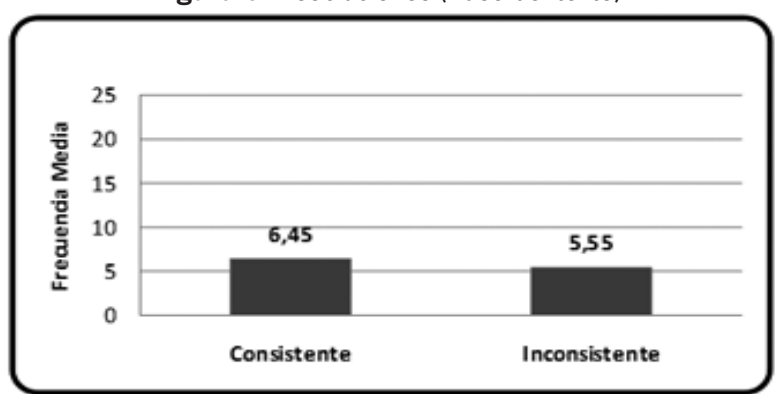

Lectura y representación mental de textos expositivos en estudiantes de educación media Myriam Cecilia Castillo Perilla / Luis Gonzalo Rodríguez López

El análisis estadístico (Anova) no presenta diferencias significativas entre la información consistente e inconsistente originada en la base del texto, ni tampoco en la proveniente del conocimiento previo.

- Inferencias explicativas. Las figuras 10 y 11 presentan las diferencias entre la información consistente e inconsistente en las inferencias asociativas. Dada la baja frecuencia de este tipo de inferencias, los análisis estadísticos no muestran diferencias significativas.

Figura 10. Explicaciones (Base de texto).

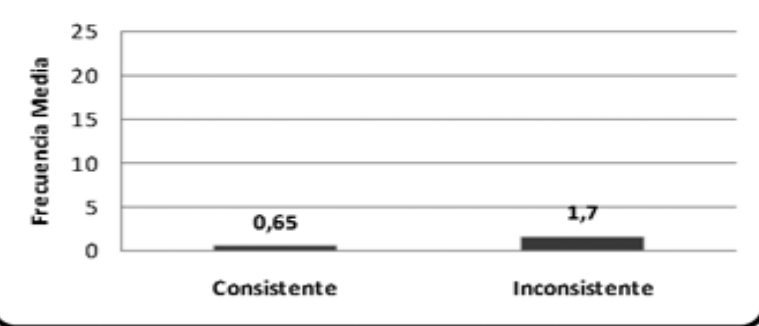

Figura 11. Explicaciones (Conocimiento previo).

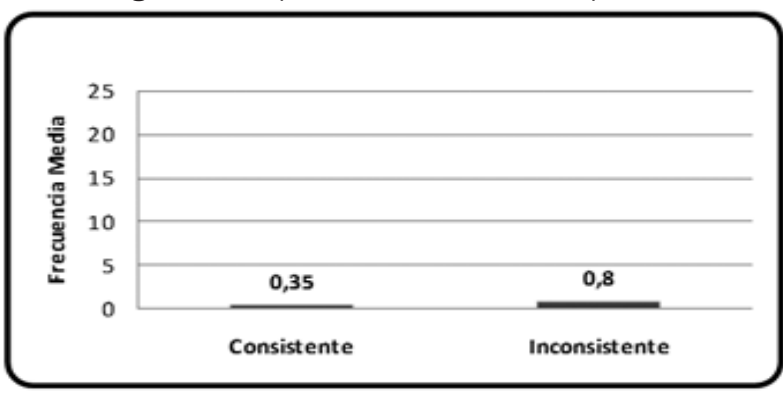

- Inferencias predictivas. De la misma manera que en las inferencias explicativas, en las inferencias predictivas la frecuencia media es reducida $y$, por tanto, los análisis estadísticos no revelan diferencias significativas (Figura 12).

Figura 12. Predicciones.

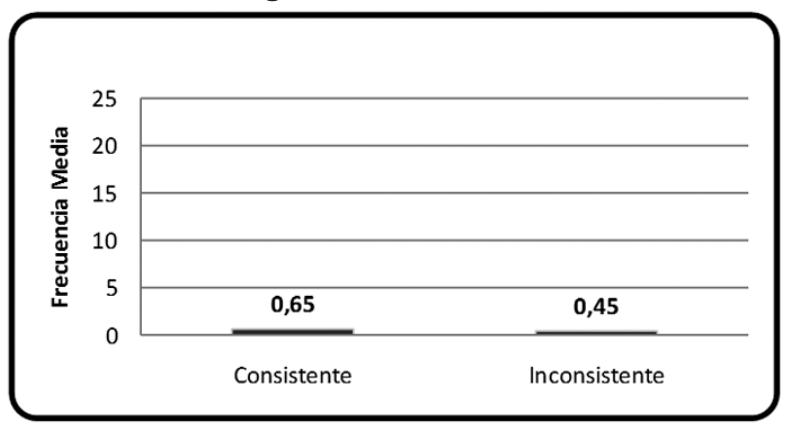


Para el caso de las evaluaciones, según se presentó en la tabla 1 y en la introducción de este análisis del modelo de situación, éstas corresponden al 7\% de la información perteneciente a este nivel de representación. A pesar de que las frecuencias son menores, el mayor número de evaluaciones se centra en el contenido (p.ej. "pero uno no tiene que ser lo mismo que está haciendo el padre") y las restantes en la tarea. No se presentaron evaluaciones de la forma del texto base (véase la figura 13).

Figura 13. Evaluaciones.

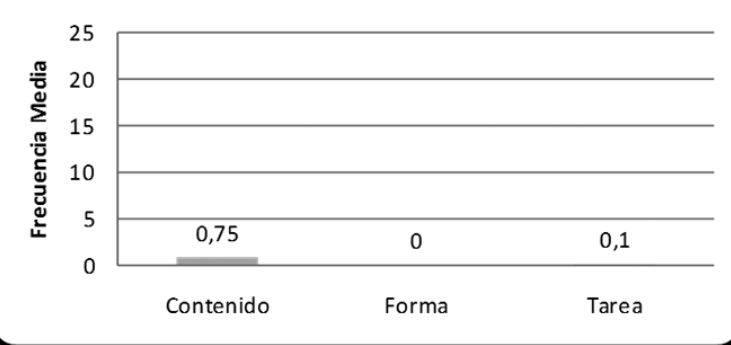

\section{Estudiantes vs expertos}

Como complemento al análisis que se hizo de las pruebas que los estudiantes presentaron, se solicitó a seis lectores expertos que subrayaran las ideas del texto que en su concepto eran las más importantes. El resultado de la tarea de los expertos arrojó que subrayaron al menos una vez 78 proposiciones distintas. En el conjunto de pruebas de los estudiantes, se hizo alusión, sumándolas todas, a 84 proposiciones del texto. Ahora bien, para efectos de adelantar este análisis, se expresaron las frecuencias de las proposiciones, tanto del subrayado de los expertos como de las proposiciones aludidas por los estudiantes, en porcentajes, para comparar los resultados (frecuencia de proposiciones $/ \mathrm{N}^{\circ}$ de participantes, 6 expertos, 20 estudiantes) y saber sobre qué proposiciones fijan su atención los dos tipos de participantes. Como punto de referencia de las proposiciones resaltadas por los expertos, se presentan aquellas que fueron subrayadas por el $66 \%$. En la tabla 3 se presentan estas proposiciones.
Tabla 3. Proposiciones más relevantes a juicio de los expertos

\begin{tabular}{|c|c|}
\hline $\mathrm{N}^{\circ}$ & Proposición \\
\hline 23 & $\begin{array}{l}\text { La evolución explica el origen de todos los seres vivos } \\
(24-25)\end{array}$ \\
\hline 24 & emparentando unos con otros (25) \\
\hline 25 & por medio de la herencia de los caracteres genéticos. \\
\hline 46 & $\begin{array}{l}\text { los seres vivos podían estar mejor adaptados al ambiente } \\
\text { [47: (Si los seres vivos) sufrían variaciones beneficiosas]. }\end{array}$ \\
\hline 65 & A este cambio accidental se le llama mutación. \\
\hline $1^{*}$ & $\begin{array}{l}\text { Desde hace cientos de millones de años, la vida sobre la } \\
\text { Tierra se ha ido haciendo más compleja. }\end{array}$ \\
\hline 22 & $\begin{array}{l}\text { Transcurridos muchos millones de años, otros se espe- } \\
\text { cializaron en vivir en un ambiente seco. }\end{array}$ \\
\hline 53 & $\begin{array}{l}\text { a lo largo de generaciones, se fueron produciendo mu- } \\
\text { chos pequeños cambios. }\end{array}$ \\
\hline 64 & $\begin{array}{l}\text { Los genes de la célula reproductora de un ser vivo varían } \\
\text { en el transcurso de su vida. (65) }\end{array}$ \\
\hline 71 & $\begin{array}{l}\text { que las mutaciones se producen normalmente en la natu- } \\
\text { raleza, una vez por cada millón de individuos. }\end{array}$ \\
\hline 73 & hay factores ambientales \\
\hline 74 & que producen mutaciones más frecuentes, (76-78) \\
\hline
\end{tabular}

La Figura 14 presenta una comparación entre las proposiciones resaltadas por los expertos y el porcentaje de las veces que los estudiantes hicieron referencia a éstas.

Figura 14. Contraste entre Expertos y Estudiantes.

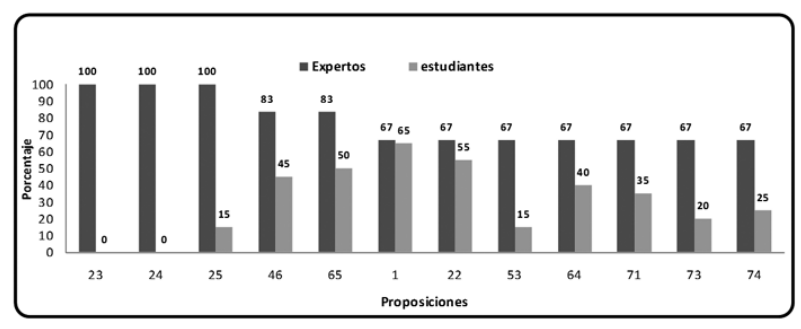

Como se puede observar, el porcentaje es bajo. La proposición en la cual hay mayor coincidencia es la $\mathrm{N}^{\circ} 1$, lo cual es explicable porque al ser la primera se conserva en la memoria durante mayor tiempo (De Vega, 1993). Esta falta de atención, por parte de los estudiantes, sobre las proposiciones importantes para los expertos, indicaría que en el proceso de comprensión aquellos no imprimen la suficiente atención a la información relevante o no logran identificarla, lo cual se evidencia en el hecho de que presentan más atención a detalles particulares y concretos, mas no a los aspectos de mayor abstracción. Con el fin de ilustrar las proposiciones 
del texto sobre las que los estudiantes fijaron más su atención, se presentan en la tabla 4 aquellas que fueron consideradas por más del 66\%.

Tabla 4. Proposiciones más relevantes a juicio de los estudiantes.

\begin{tabular}{|c|c|}
\hline $\mathrm{N}^{\circ}$ & Proposición \\
\hline 20 & tanto en la tierra \\
\hline 38 & $\begin{array}{l}\text { (Por eso) Un animal al que le falte una pata no tiene por } \\
\text { qué producir descendientes sin esa pata }\end{array}$ \\
\hline 21 & como en el agua. \\
\hline 12 & $\begin{array}{l}\text { Algunos (organismos pluricelulares) adquirieron la posibili- } \\
\text { dad de flotar, como las medusas. }\end{array}$ \\
\hline 37 & $\begin{array}{l}\text { que las variaciones corporales no pueden heredarse de } \\
\text { padres a hijos. }\end{array}$ \\
\hline 19 & estos animales adquirieron la posibilidad de vivir (20-21) \\
\hline 30 & $\begin{array}{l}\text { Según la primera teoría, las características adquiridas } \\
\text { a lo largo de la vida se heredan por sus descendientes } \\
\text { (31-33) }\end{array}$ \\
\hline 9 & (Así) Se formaron los primeros organismos pluricelulares, \\
\hline 15 & sencillos órganos, semejantes a las aletas. \\
\hline 17 & $\begin{array}{l}\text { en el transcurso de muchos millones de años ciertos } \\
\text { animales que vivían en las proximidades de la tierra se } \\
\text { adaptaron a vivir en ella. }\end{array}$ \\
\hline 31 & (teoría) propuesta por Lamarck \\
\hline $1^{*}$ & $\begin{array}{l}\text { Desde hace cientos de millones de años, la vida sobre la } \\
\text { Tierra se ha ido haciendo más compleja. }\end{array}$ \\
\hline 34 & La teoría de Lamarck se ha abandonado \\
\hline
\end{tabular}

En la Figura 15 se muestran las proposiciones que presentan mayor porcentaje en los resultados que se obtuvieron de los ejercicios de los estudiantes, y el porcentaje de mención hecho por los expertos para las mismas.

Figura 15. Contraste entre Estudiantes y Expertos.

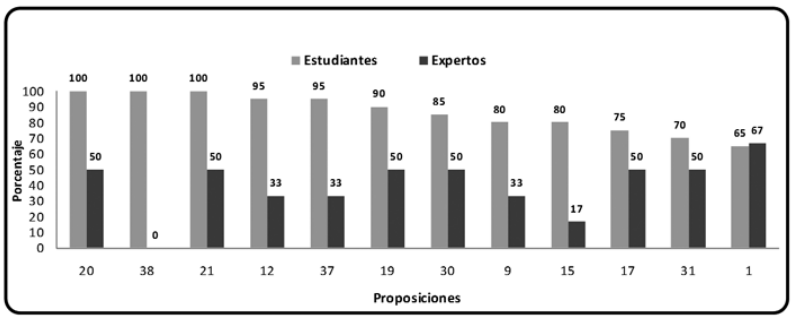

\section{Discusión}

Los resultados del análisis de las representaciones mentales de los estudiantes reflejan que la información no está distribuida de manera equilibrada en los niveles base del texto $(4,05)$ y modelo de situación $(23,05)$. Este hecho refleja cómo el proceso
Lectura y representación mental de textos expositivos en estudiantes de educación media Geral Eduardo Mateus Ferr Myriam Cecilia Castillo Perilla / Luis Gonzalo Rodríguez López

de lectura integra tanto la información proveniente del texto como la aportada por el sujeto lector. En este caso, la representación mental presenta más información perteneciente al sujeto que originada en el texto.

Sin embargo, se halló que los estudiantes aportan información consistente e inconsistente a su representación. Este hecho refleja que la comprensión de las ideas expresadas en el texto es deficiente y/o que los conocimientos sobre el tema no son los adecuados, aspecto que incide de manera significativa en el proceso lector. Sin embargo, este hecho no es inusual; al respecto, Otero y Campanario (1990) y Otero y Kintsch (1992), en sus estudios sobre comprensión de textos con contenidos científicos que incluían contradicciones explícitas, descubrieron que es frecuente que los estudiantes construyan explicaciones, evidentemente paradójicas, en las que concilian las contradicciones. En tal sentido, no es extraño que las representaciones de los estudiantes en la prueba de recuerdo hayan integrado información con distintos grados de consistencia. Por ejemplo, un estudiante escribió "que Lacnaks [sic] se equivocó en su teoría porque decía que el hijo podía heredar las capacidades de su padre, ejemplo si el padre era un corredor el hijo también lo podía ser). Si bien el texto sugiere que la teoría se abandonó, y el estudiante infiere que se "equivocó", lo cual puede considerarse como consistente, la explicación de la supuesta equivocación es inconsistente, pues en el texto se menciona la incapacidad para heredar variaciones corporales, no la de habilidades (p. ej. ser corredor). Aún así, el estudiante integra las informaciones sin notar sus inconsistencias.

Con respecto a las diferencias entre los tipos de inferencias, vale la pena retomar a Narváez et al (1999), quienes en su estudio con protocolos de pensamiento en voz alta ("think aloud protocols") encontraron un mayor número de inferencias asociativas $(0,04)$ sobre las explicativas $(0,004)$ y las predictivas $(0,002)$. Este antecedente se replica en los resultados que se obtuvieron en la tarea de recuerdo libre que se utilizó (ver Figura 3), que muestra que las inferencias asociativas, es decir, aquellas que dan cuenta de las características, propiedades y relacio- 
nes de los objetos, personas y conceptos, son las de mayor frecuencia, seguidas por las explicativas (inferencias hacia atrás) y las predictivas (inferencias hacia adelante).

Los estudiantes construyen gran parte de su modelo mental añadiendo información sobre las entidades (p. ej. "los peces que desarrollaron aletas para movilizarse en el agua"). En el texto no se mencionan explícitamente "los peces", pero a través de una asociación el estudiante vincula "los organismos" indicados en el texto con "peces", acudiendo a su conocimiento previo: "los peces tienen aletas".

Es menor la creación de inferencias explicativas. Una muestra de éstas es: "Porque, si el [animal] (sic) no está en su hábitat podría sufrir [...]”. Se establece una relación causal entre el hábitat y el ser vivo. Ahora bien, y también consistente con los antecedentes en estudios sobre inferencias, las inferencias predictivas son las más escasas. He aquí un ejemplo de las creadas por los estudiantes: "[Algunas especies al adaptar sus genes] podrán cambiar la textura de su pelo". En esta inferencia se proyecta la situación futura del aspecto de algunas especies, a partir de las posibilidades de adaptación sugeridas en el texto base.

De otra parte, en cuanto a las diferencias con los lectores expertos, como se puede apreciar en los resultados (ver Figura 15), el porcentaje de coincidencia, salvo en un caso, es bajo. Este aspecto estaría relacionado con el hecho de que los expertos centran su atención en los aspectos más generales y abstractos, esto es, las ideas básicas que en el texto se presentan, dejando de lado los detalles, los datos concretos y las ejemplificaciones, elementos que, por el contrario, se encuentran en mayor medida en las representaciones mentales de los estudiantes; asimismo, dejan por fuera aspectos relevantes para la comprensión del texto. En este sentido, se podría plantear que los estudiantes presentan dificultades al momento de deslindar lo que se considera como ideas principales, o afirmaciones, de aquello que forma parte del texto pero que es prescindible. Desde luego, esta dificultad está asociada a factores como el tipo de texto, los conocimientos previos del lector, el tipo de tarea, los intereses del individuo, entre otros; no obstante, se constituye también en razón para plantear opciones didácticas que se sustenten en los planteamientos de la psicología discursiva, con el fin de desarrollar la capacidad lectora de los educandos.

No obstante, si se retoman las características del modelo de situación enunciadas previamente, efectivamente hay coincidencias entre expertos y estudiantes. A pesar de que la tarea sugerida a los expertos no implica la construcción de un modelo de situación, sí resaltan ideas que pueden hacer parte de aquel. Por ejemplo, tanto los expertos como los estudiantes hacen alusión a referencias espaciales y temporales, lo cual es típico de los modelos de situación, en la medida en que éstos retienen aspectos de las interacciones senso-motoras que los individuos experimentan en relación con las situaciones que se presentan en el texto.

Ahora bien, volviendo al contraste entre la información señalada por unos y otros, a diferencia de los datos obtenidos de los expertos, en las proposiciones que los estudiantes retoman del texto se encuentran más conceptos particulares que en el texto se presentan como ejemplos de casos generales (p. ej. medusas, pata, aletas), aspecto que permite señalar nuevamente que los estudiantes se fijan más en los detalles concretos que en las generalidades, por lo que se hace más difícil identificar las ideas básicas del texto y, a la vez, recordarlas.

En general, en lo que respecta al estudio de las representaciones mentales, se ha evidenciado que los aportes de la psicología de la comprensión son aplicables al estudio del proceso de lectura en el campo educativo. La tradición de los estudios en la perspectiva psicológica y cognitiva permite dar cuenta de la lectura como proceso complejo, que implica la intervencion de tareas tales como el almacenamiento y la retención de información en las estructuras de la memoria (De Vega, 1993), la consecuente representación de la información en distintos niveles y su activación e integración con conocimientos previos.

El análisis proposicional se presenta como un recurso útil para identificar los componentes de las representaciones, dada su posibilidad diferenciadora 
como la consecuente posibilidad de cuantificación (De Vega et al, 1990). La decisión de no tomar como unidad de análisis una de carácter exclusivamente formal lingüístico (p. ej. la oración), y acudir a una de carácter psicológico (i.e. la proposición), que no desconoce lo lingüístico, se presenta como una vía que intenta resolver la dicotomía entre forma y significado. Además, la noción de proposición, asociada a la de entender el resultado del proceso lector, en términos psicológicos, como una "representación mental", permite centrarse en los procesos mentales implicados en tal tarea. Obviamente, reconociendo que la lectura también tiene implicaciones en otros órdenes (p. ej. social, pragmático).

En cuanto a la caracterización como tal de las representaciones mentales de los estudiantes, se puede plantear que en éstas priman los componentes del modelo de situación sobre la información que procede directamente de la base del texto. A su vez, se determinó que el modelo de situación está integrado, en su mayoría, por inferencias asociativas, seguidas por explicativas y en menor número predictivas; aspecto este que es consistente con los resultados obtenidos por otros trabajos (p. ej. Narváez et al, 1999).

De manera consistente con los antecedentes, y con las teorías que plantean que la representación mental generada al comprender textos equivale a una entidad mental compleja, que integra tanto la información procedente del texto como tal con la aportada por el sujeto lector (De Vega y Cuetos, 1999; De Vega, Díaz y León, 1999; León, 2002; Graesser, Millis y Zwaan, 1997; Kintsch, 1994; Kintsch y van Dijk, 1983; Narváez et al, 1999), los resultados de la prueba de recuerdo que se tomaron como fuente para analizar la representación mentalde los estudiantes ratifican estos planteamientos, en la medida en que se evidencian tanto elementos del texto de base como del conocimiento del individuo (transformándolos del texto base o tomándolos del conocimiento previo) que se presenta en el modelo de situación.

Si se considera que el propósito final del proceso de compresión lectora es reconstruir el significado de un texto, y que para ello es necesaria la interac-
Lectura y representación mental de textos expositivos en estudiantes de educación media Myriam Cecilia Castillo Perilla / Luis Gonzalo Rodríguez López

ción de los conocimientos del lector con la información provista por los textos, se puede afirmar que el grupo de estudiantes al que se aplicó la prueba presenta dificultades en la comprensión, pues integran un porcentaje considerable (aproximadamente un 50\%) de información inconsistente en sus representaciones; asimismo, se evidenció que presentan dificultades para separar las ideas básicas del texto de las informaciones incidentales. En tal sentido, es necesario cualificar sus procesos de lectura; por ello se hace, en principio, pertinente la formulación de una propuesta didáctica que se fundamente en este tipo de modelos y que recurra a aspectos como la metacognición para potenciar la capacidad lectora de los individuos.

\section{Referencias}

Aznar, J. (1990). Pruebas de memoria: estructura formal, comportamientos y estrategias. En Anuario de psicología, 45, 43-63. Barcelona: Universidad de Barcelona.

De Vega, M. (1993). Introducción a la psicología cognitiva. (6 ${ }^{\text {a }}$ reimpresión). Madrid: Alianza Editorial.

De Vega, M. et al, (1990). Lectura y comprensión. Una perspectiva cognitiva. Madrid: Alianza Editorial.

De Vega, M. y Cuetos, F. (Coords.). (1999). Psicolingüística del español. Madrid: Trotta S.A.

De Vega, M., Díaz, J. y León, I. (1999). Procesamiento del discurso. En M. De Vega y F. Cuetos (Eds.), Psicolingüística del español (pp. 271-306). Madrid: Trotta.

Graesser, A., Millis, K. \& Zwaan, R. (1997). Discourse Comprehension. In Annual Review of Psychology, 48, 163-189.

Kintsch, W. (1994). Text Comprehension, Memory, and Learning. In American Psychologist, 49(4), 294-303.

Kintsch, W. \& Van Dijk, T. (1983). Strategies of Discourse Comprehension. New York: Academic Press.

León, J. (2002). Conocimiento y discurso: claves para inferir y comprender. Madrid, Pirámide.

León, J., Escudero, I. y Van Den Broek, P. (2003). La influencia del género del texto en el establecimiento de inferencias elaborativas. En J. León (Coord.), Conocimiento y discurso. Claves para inferir y comprender. Madrid: Pirámide. 
Montanero, M. (2004). Cómo evaluar la comprensión lectora: alternativas y limitaciones. En Revista de educación, 335, 415-427.

Narváez, D., Van den Broek, P. \& Ruiz, A. (1999). Reading Purpose, Type of Text and Their Influence on ThinkAlouds and Comprehension Measures. In Journal of Educational Psychology, 91(3), 488-496.

Otero, J. \& Campanario J. (1990). Comprehension Evaluation and Regulation in Learning from Science Texts. In Journal of Research in Science Teaching, vol. 27(5), 447-460.

Pérez, M. (2005). Evaluación de la comprensión lectora: dificultades y limitaciones. En Revista de educación, número extraordinario, 121-138.
Pinzás, J. (2003). Metacognición \& lectura. Lima: Pontificia Universidad Católica del Perú.

Restrepo, B. et al (2004). Investigación-acción educativa: una estrategia de transformación de la práctica pedagógica de los maestros. Bogotá: Aguilar.

Vidal-Abarca, E., Gilabert, R. y Abad, N. (2002). Una propuesta para hacer buenos textos expositivos: hacia una tecnología del texto expositivo. En Infancia y Aprendizaje, 24(4), 499-514.

Zwaan, R. \& Brown, C. (1996). The Influence of Language Proficiency and Comprehension Skill on SituationModel Construction. In DiscourseProcesses, 21, 289-328.

\section{Anexo}

Análisis de las proposiciones* del texto "La evolución de las especies".

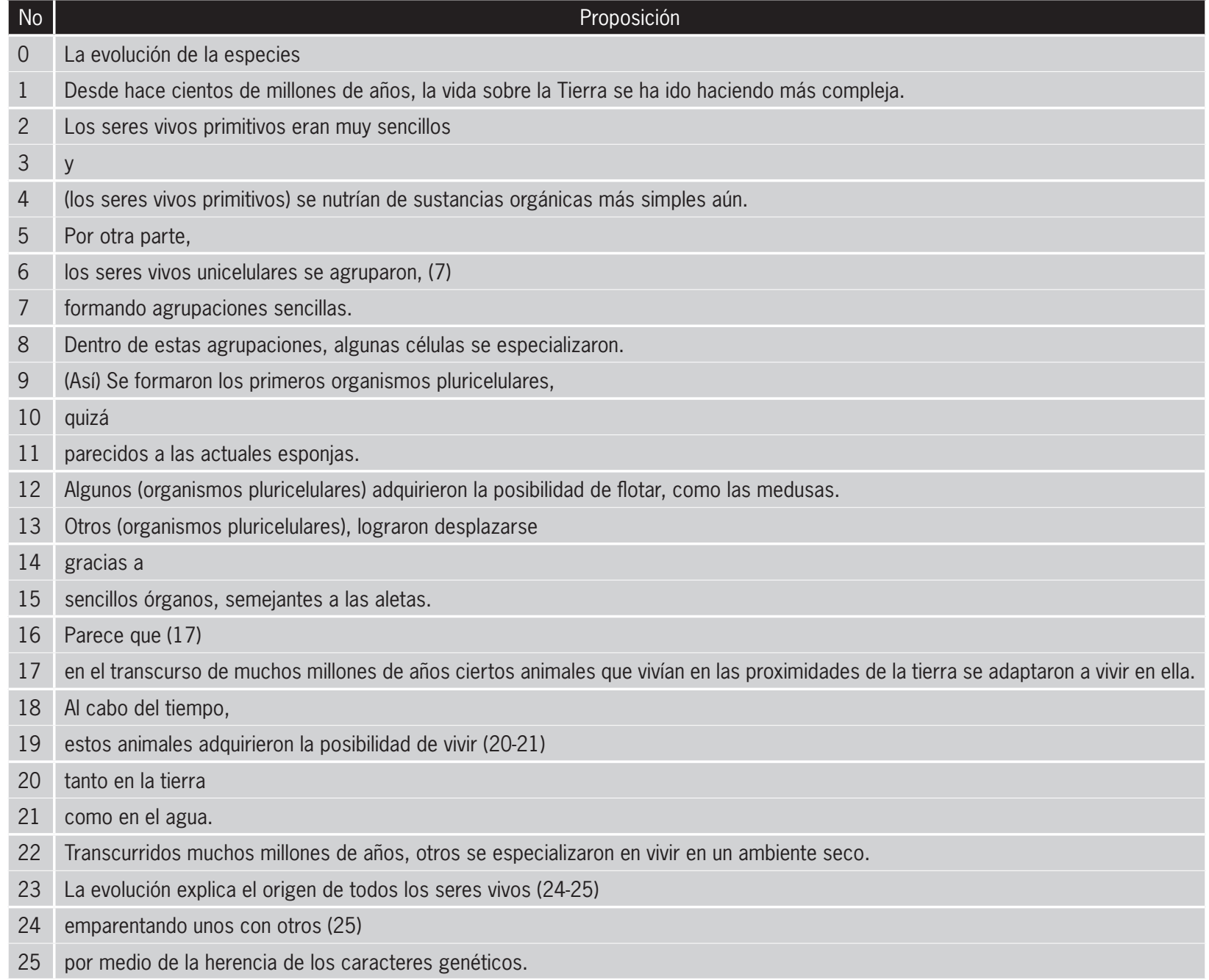


26 Las primeras observaciones sobre la posible evolución de los seres vivos proceden de los antiguos griegos.

27 Sin embargo,

28 hasta hace más de un siglo, los biólogos no se atrevieron (29)

29 a formular ninguna teoría sobre la evolución.

30 Según la primera teoría, las características adquiridas a lo largo de la vida se heredan por sus descendientes (31-33)

31 (teoría) propuesta por Lamarck

32 por ejemplo,

33 los músculos desarrollados por un atleta

34 La teoría de Lamarck se ha abandonado

35 porque

36 se demostró

37 que las variaciones corporales no pueden heredarse de padres a hijos.

38 (Por eso) Un animal al que le falte una pata no tiene por qué producir descendientes sin esa pata

39 La teoría admitida actualmente fue propuesta por Darwin a mediados del siglo pasado.

40 Darwin observó (41)

41 que en todos los seres vivos se producían variaciones en su estructura.

42 Algunas (variaciones) eran perjudiciales para el individuo

43 pero

44 otras(variaciones) eran favorables.

45 Darwin propuso (46)

46 que los seres vivos podían estar mejor adaptados al ambiente (47).

47 (Si los seres vivos) sufrían variaciones beneficiosas

48 Estos seres vivos tenían más posibilidades de tener una larga vida

49 (tenían más posibilidades) de reproducirse

$50 \mathrm{y}$

51 (tener más posibilidades) de transmitir las variaciones genéticas.

52 Según Darwin,

53 a lo largo de generaciones, se fueron produciendo muchos pequeños cambios.

54 Si estos cambios facilitaban la supervivencia,

55 (entonces) se originaban individuos con caracteres distintos de los caracteres que tenían sus lejanos antecesores.

56 Durante la reproducción, (57) resultan nuevos organismos de características genéticas distintas a las de sus progenitores.

57 (esto sucede) al formarse los cromosomas de las células sexuales

58 Así se originan nuevos organismos, (59-63)

59 (organismos que son) todos distintos entre sí,

$60 \mathrm{y}$

61 (organismos que pueden estar) mejor (adaptados al ambiente)

620

63 peor adaptados al ambiente.

64 Los genes de la célula reproductora de un ser vivo varían en el transcurso de su vida. (65)

65 A este cambio accidental se le llama mutación.

66 En algunos casos, esta deficiencia es grave

$67 \mathrm{y}$

68 (esta deficiencia) puede llegar a producir la muerte del individuo.

69 En unos pocos casos, la mutación origina un individuo con mejores características.

70 Se calcula (71) 
71 que las mutaciones se producen normalmente en la naturaleza, una vez por cada millón de individuos.

72 Pero

73 hay factores ambientales (74)

74 que producen mutaciones más frecuentes, (76-78)

75 por ejemplo,

76 ciertas sustancias químicas

770

78 la radioactividad.

79 Imaginemos (80)

80 que el proceso de la evolución actúa sobre una población de ovejas de una región fría.

81 En esta población una oveja podría sufrir una variación (favorable en el gen responsable de la producción de lana) (82)

820

83 una mutación (5) favorable en el gen responsable de la producción de lana.

84 (Así) Esta oveja estaría mejor adaptada que el resto (85)

85 para resistir el frío.

86 (Por lo tanto) La oveja sobreviviría un mayor número de estaciones

$87 \mathrm{y}$

88 podría producir más descendientes que otra del rebaño.

89 (En consecuencia) Aparecerán ovejas mejor adaptadas que el resto de la población.

90 (Como resultado) Al cabo de mucho tiempo se originará una variación en la especie

$91 \mathrm{y}$

92 aumentará cada vez más el número de ovejas que poseen ese determinado gen.

93 (De esta forma) Se habrá producido una selección de la especie.

94 Al cabo del tiempo, las ovejas menos adaptadas pueden llegar a desaparecer. 